\title{
Empresas recuperadas por cooperativas
}

\author{
M. Eleonora Feser \\ Universidad Nacional de Moreno. Universidad de Buenos Aires, Facultad de Derecho \\ mfeser@gmail.com
}

Sumario: 1. Introducción.-2. La recuperación de empresas en Argentina, el camino hacia el cooperativismo.-3. Concepto de ERT desde un enfoque jurídico.- 4. Particularidades en materia legal, fiscal y de la seguridad social. 4.1 Organismo estatal de promoción y control. Datos desde el Estado. 4.2 Financiamiento. 4.3 Regulación fiscal. 4.4 Seguridad social.-5. Reflexiones preliminares. Referencias

Resumen: La recuperación de empresas por cooperativas de trabajo ha sido un fenómeno que se ha forjado no solo desde la práctica sino también por la normativa que atraviesa parte de estos procesos.

En el presente trabajo se analiza cómo ha sido este camino desde lo legal, haciendo foco en el momento histórico en el que aparecen las empresas recuperadas por sus trabajadores (ERTs) en la ley de concursos y quiebras y su exigencia de conformarse bajo la figura de cooperativa de trabajo. También se realizará un mapeo a nivel nacional, provincial y municipal de toda la normativa donde se menciona a las empresas recuperadas, categorizándola de manera tal de permitir un análisis. Finalmente se abordarán otras particularidades a nivel regulación.

Palabras clave: Empresas Recuperadas. Autogestión. Marco legal. Argentina.

Abstract: The recovery factories by worker cooperatives has been a phenomenon that has been forged not only by practice but also by the regulations that have been part of these processes.

This paper analyzes the legal aspects of this process, focusing on the historical moment in which the worker-recuperated enterprises (ERTs) appear in the bankruptcy law and its requirement to be established as worker cooperatives. A mapping will also be made at the national, provincial and municipal levels of all the regulations that mention recovery factories, categorizing them in such a way as to allow an analysis. Finally, other particularities at the regulatory level will be addressed.

Keywords: Recovery factories. Cooperatives. Legal Framework. Argentina. 


\section{Introducción}

El presente trabajo de corte preliminar intenta realizar una búsqueda exploratoria que contribuya al análisis del fenómeno de la recuperación de empresas por sus trabajadores (en adelante ERT) en manos de Cooperativas de Trabajo.

El cooperativismo como sistema de organización social, política y económica es una alternativa al sistema capitalista, en definitiva, una respuesta integradora a las problemáticas que se oponen a la visión imperante de las corrientes liberales y neoliberales que intentan disociar la economía de la política, del dominio de lo social, naturalizando las relaciones de poder imperantes.

A mediados del siglo XIX emergen los primeros movimientos cooperativos basados en la cooperación en el consumo, debido a los precios inaccesibles de los bienes de consumo popular, una respuesta a la carestía de la vida. En la Argentina, a principios del siglo Xx las primeras corrientes migratorias provenientes en su gran mayoría de Europa Occidental, no solo traían sus ansias de progreso, sino, también un modelo que encontró asidero en un país pujante pero profundamente desigual, dando pie a las primeras cooperativas de consumo.

En el año 1906, luego de la suba de los precios de alquileres en los grandes centros urbanos derivó en la primera huelga de inquilinos, frente a ello, la cooperación en la construcción y autoconstrucción de viviendas populares vio sus cimientos. En la segunda década del mismo siglo, la necesidad de establecer mecanismos de crédito no usurario devino en las primeras cajas, cooperativas de crédito y banca solidarias, sumado a ello, diversas experiencias de organización de las comunidades en distintas geografías dieron nacimiento a la cooperación en la prestación de servicios públicos.

El trabajo, elemento integrador de la sociedad, también ha transitado transformaciones, avances y retrocesos, en las épocas de crisis de empleo, el cooperativismo ha sido una respuesta a las relaciones del trabajo dominantes envueltas en el binomio capital-trabajo, cuenta de ello, a fines del siglo XX y XXI se expresa el cooperativismo de trabajo como una contestación a las crisis económicas generadas por el modelo económico, social y político neoliberal.

En Argentina, las políticas económicas, sociales y sobre todo las de la última década del siglo $\mathrm{xx}$, fundamentalmente de apertura indiscriminada de importaciones, derivaron en una profunda crisis, deteriorando el tejido social y la caída de la actividad económica y como consecuencia la reducción del aparato productivo, dándose origen a una estrategia de acción colectiva proveniente de los trabajadores quienes 
ocupaban las empresas en búsqueda de la recuperación de su fuente de trabajo, dando origen a las «Fábricas Recuperadas por sus trabajadores» (en un principio mayoría fabriles), para luego incluir otras actividades construyendo el colectivo de ERTs.

En este orden de ideas, el análisis realizado en el presente trabajo consta de tres partes, no entendidas como compartimentos estancos, más si interrelaciones, que se retroalimentan mutuamente para esbozar brevemente la regulación de las Empresas Recuperadas por cooperativas y sus particularidades legales en la Argentina. En primer término se analizará desde el derecho positivo como se ha forjado el vínculo entre el cooperativismo de trabajo y las ERTs. La primera herramienta utilizada ha sido la Ley de Concursos y Quiebras n. ${ }^{\circ} 24.522$ (en adelante LCQ) y los cambios de paradigma introducidos respecto de su antecesora. La Ley $n .^{\circ} 26.684$, el contexto donde irrumpen estos procesos y la manera en que lo han hecho.

Inmediatamente se avanza sobre el concepto de ERT desde un enfoque jurídico, a través de la recolección de datos que se desprenden de un mapeo de las normas a nivel nacional, provincial ${ }^{1}$ y municipal. Para realizar esta tarea se partió de las palabras clave "empresa recuperada» utilizando el repositorio jurídico de normas de Thomson Reuters. Con el total de elementos relevados se confeccionó una tabla que contiene inicialmente 46 elementos. Se establecieron diversas categorías de clasificación y análisis según el tipo de norma de que se trate, año de su sanción, jurisdicción y el abordaje al concepto de ERT o si al menos simplemente se la nombra. Este análisis nos permitirá conocer como el derecho positivo conforma la definición de empresa recuperada y su vínculo con el cooperativismo.

La última parte de este trabajo, analiza brevemente las particularidades de la utilización de la figura cooperativa en la recuperación de empresas. Para ello se hará un breve recorrido relacionado con la autoridad de aplicación de la ley de cooperativas y su especificidad en lo que a ERT refiere. Por otro lado se avanzará en una referencia respecto a programas estatales diseñados para atender el financiamiento de estas experiencias. Se detallarán aspectos relacionados con la tributación en términos generales y particulares de las ERTs en su forma cooperativa para finalizar esta parte con un breve análisis en materia de seguridad social, siendo este uno de los aspectos

1 A los efectos de simplificar el análisis y su lectura, se consideró a la Ciudad Autónoma de Buenos Aires dentro de la categoría «Ley provincial» aunque en realidad se trate de una Ciudad Autónoma. 
donde más desafíos encuentran estas experiencias dentro del cooperativismo.

Por último, se plantean algunos puntos a través de reflexiones finales respecto a la hipótesis de la potencialidad de la recuperación de empresas por cooperativas de trabajo así como los desafíos que tiene el movimiento cooperativo al abrazar estos procesos autogestionados y la posibilidad de replicarlas en otros territorios.

\section{La recuperación de empresas en Argentina, el camino hacia el cooperativismo}

La elaboración, discusión, sanción y promulgación de una norma es antecedida por un hecho social. La misma conlleva la institucionalización de un estatus identitario, el reconocimiento del Estado, el que detenta para sí el poder formal de crear, regular, modificar, relaciones jurídicas y aplicar el monopolio de la fuerza legítima en caso de incumplimiento. En términos de Coraggio (2014), se definen dos categorías de institucionalización: ampliada y restringida, siendo la primera la asociación a la cultura, al sentido común, al habitus, desde la perspectiva de Bourdieu, es decir, la incorporación de prácticas sociales determinadas y, por otro lado, el concepto restringido o limitado, que implica la anexión y creación de institutos jurídicos, estructuras políticas, administrativas y burocráticas, que resignifiquen y relacionan principalmente con el Estado (Chiesa, 2021).

Como primera cuestión característica nos debemos preguntar ¿Qué es una ERT? Según lo expuesto por Facultad Abierta (2014): "se trata de una unidad económica - productiva o de servicios- que atraviesa un proceso por el cual pasa de la gestión privada a la gestión colectiva de sus antiguas/os asalariadas/os». Si bien esta definición otorga diversos elementos de análisis nada dice respecto a la forma jurídica adoptada.

En lo que refiere al proceso en la Argentina, cada caso en particular tiene una riqueza histórica y una complejidad individual, aunque sea posible encontrar regularidades que los contengan a todos (Facultad Abierta, 2004), en este sentido, la denominada LCQ es referencia de gran parte de los procesos relevados, aunque no la totalidad de ellos, por el hecho de expresarse en el marco de procesos concursales, a saber empresas con dificultades económico financieras donde los propios dueños o terceros que le solicitan la quiebra judicialmente. A partir de dichos procesos la norma fundamental que los regula y reglamenta a nivel federal es la denominada ley falencial. 
Cierto es que la LCQ, ha transitado diversas transformaciones al calor de los hechos. Históricamente, al decretarse la resolución que determinaba la quiebra de una persona humana y/o jurídica, el principal y único objetivo del juez concursal era la pronta satisfacción de los créditos, frente a ello la justicia debía poner en marcha todo su funcionamiento teniendo en miras la pronta realización de los bienes y poder así, de modo alguno, satisfacer los créditos verificados en el proceso. Pero esto no siempre fue así, en otros momentos históricos la LCQ tenía por objetivo la conservación de la empresa, por lo que se generaban desde la normativa determinadas herramientas que permitieran cumplir con este objetivo. Existen numerosos trabajos que realizan esta historicidad, como Maffia (1995), Farhi de Montalban (2005), Favier Dubois (h.) (2011), entre otros.

Es así como entre dichas disputas, «los pro liquidación vs los pro conservación», ha navegado la normativa falencial en la Argentina, modificándose acorde a los lineamientos de la política nacional en cada contexto histórico. Muestra de esto es la regulación de la ley falencial en los años 90, donde predominaba un claro corte neoliberal, en el que ciertos institutos fueron eliminados, como el de la calificación de la quiebra y otros fueron incorporados, como el mal llamado cramdown $\mathrm{o}$, en su correcta interpretación, el salvataje empresarial, en donde el Juez puede avanzar en un acuerdo aún cuando no se hayan alcanzado las mayorías legales necesarias, o la introducción del denominado APE, Acuerdo Preventivo Extrajudicial, donde la persona (ya sea humana o jurídica) que se encuentra con dificultades financieras, puede acudir a este procedimiento extrajudicial, acordar con ciertos acreedores y homologar en la justicia dicho acuerdo haciéndolo extensivo a aquellos que no participaron en la negociación.

En virtud de la profunda crisis atravesada por nuestro país en el año 2001, en donde la Argentina se encontraba en un contexto socioeconómico complejo, el país había sufrido una gran crisis social, económica y política que implosionó en las represiones del 19 y 20 de diciembre del año 2001, la renuncia del presidente Fernando de la Rúa y una seguidilla de cinco presidentes en una semana. Un contexto de desempleo que alcanzaba el $18 \%$ y de trabajo informal del $38 \%$, la mirada social respecto al valor trabajo se encontraba en su auge, por lo que el proceso de recuperación de empresas y su acompañamiento normativo era algo esperable (Rebón, 2007).

En el año 2002 se produce una reforma de la LCQ irrumpiendo, ahora si las ERTs en una norma hasta ese momento no pensada ni por ni para ese proceso particular. 
Dicha aparición no fue pacífica ni integral, simplemente se limitó a incorporar, en una ley creada y pensada en el año 1995, un artículo 2 que diera cuenta de la existencia de dicho fenómeno y algunas pequeñas pautas, dejando abiertos ciertos aspectos que luego serían completados por los tribunales, en un territorio de disputas de sentidos y miradas que decantaría en una reforma mayor 9 años después.

En ese único artículo incorporado en el año 2002, se mencionó la posibilidad de «tomar en consideración» el pedido formal de los trabajadores, por lo que no era una obligación, sino una proposición para el juez. A su vez, para acceder a ese pedido, los trabajadores debían contar con un mínimo de créditos laborales particular, y con una forma jurídica específica, la Cooperativa de Trabajo.

Como se mencionó, 9 años luego, a saber el 29 de junio de 2011 se promulgó una nueva reforma concursal mediante la Ley 26.684, necesaria para recuperar las experiencias recogidas por los tribunales en casos concretos (Junyent Bas, 2011) y con ella numerosos avances que, permitieron a los trabajadores brindarles un marco normativo, que los reconociera como continuadores de la explotación de la empresa fallida entre otros avances que se fueron generando a lo largo de las experiencias recogidas en los tribunales.

2 El artículo mencionado es el 190 que dice «En toda quiebra, aun las comprendidas en el artículo precedente, el síndico debe informar al juez dentro de los veinte (20) días corridos contados a partir de la aceptación del cargo, sobre la posibilidad excepcional de continuar con la explotación de la empresa del fallido o de alguno de sus establecimientos y la conveniencia de enajenarlos en marcha.

En la continuidad de la empresa se tomará en consideración el pedido formal de los trabajadores en relación de dependencia que representen las dos terceras partes del personal en actividad o de los acreedores laborales quienes deberán actuar en el período de continuidad bajo la forma de una cooperativa de trabajo.

El término de la continuidad de la empresa, cualquiera sea su causa, no hace nacer el derecho a nuevas indemnizaciones laborales.

El informe del síndico debe expedirse concretamente sobre los siguientes aspectos: 1) La posibilidad de mantener la explotación sin contraer nuevos pasivos; 2) La ventaja que resultaría para los acreedores de la enajenación de la empresa en marcha; 3) La ventaja que pudiere resultar para terceros del mantenimiento de la actividad; 4) El plan de explotación, acompañado de un presupuesto de recursos, debidamente fundado; 5) Los contratos en curso de ejecución que deben mantenerse; 6) En su caso, las reorganizaciones o modificaciones que deben realizarse en la empresa para hacer económicamente viable su explotación; 7) Los colaboradores que necesitará para la administración de la explotación; 8) Explicar el modo en que se pretende cancelar el pasivo preexistente.

El juez a los efectos del presente artículo y en el marco de las facultades del artículo 274, podrá de manera fundada extender los plazos que se prevén en la ley para la continuidad de la empresa, en la medida que ello fuere razonable para garantizar la liquidación de cada establecimiento como unidad de negocio y con la explotación en marcha.» 
Un primer efecto que tuvo la reforma de la ley se produjo en relación a la utilización de las expropiaciones como modos de frenar procesos de venta de bienes en la quiebra. Es así como a partir de su sanción en el año 2011 se disminuyó significativamente la utilización de dicho mecanismo, en buena parte porque a partir de la inclusión de la posibilidad de la cooperativa de continuar con la unidad económica bajo reglas en principio más claras (Facultad Abierta, 2014).

Otro efecto de la reforma, fue intentar modificar ese paradigma de «la pronta liquidación de los bienes» (Tévez, 2011), para pasar a uno nuevo que tenga en miras al sujeto trabajador como principal damnificado frente a una situación de crisis empresarial, y con ello la necesidad imperiosa de conservar su fuente de trabajo, como sustento familiar, de vida y de dignidad de cualquier persona, poniendo como centro la reproducción del hombre tal como lo hace el cooperativismo.

De allí que esta forma jurídica adquirió, por lo menos para una parte de las recuperaciones, tal como veremos en los siguientes apartados, un cuerpo y dirección particular. Los trabajadores, si querían hacer uso de esta herramienta indefectiblemente deberían elegir esa forma jurídica, aunque su realidad comercial les exigiera otra cosa ${ }^{3}$.

Más allá de lo mencionado, es cierto que de las posibilidades existentes en la Argentina en ese momento, la cooperativa de trabajo es la que más parece ajustarse a las realidades del colectivo. Se reconocía que el trabajo bajo la figura empleador-empleado había colapsado hacia nuevas formas autogestionadas donde todos los trabajadores se encontraban en un pie de igualdad para enfrentar cuestiones vinculadas a la toma de decisiones hasta en lo que hace a aspectos relacionados con lo económico, por lo que los principios, valores y caracteres de las cooperativas permitían materializar esta igualdad. Por ejemplo, el principio de un socio-un voto propio del cooperativismo, así como la eliminación de intermediarios y la retribución en función al uso de la figura, fueron aspectos claves para hacer del cooperativismo la mejor experiencia posible para contener y acompañar procesos tan complejos como es la recuperación de una empresa en manos de sus trabajadores.

El espíritu de las reformas de 2002 y 2011 de la LCQ han sido y seguirá siendo un instrumento de disputas en el derecho comercial, abriéndose en cada proyecto de ley el debate entre aquellos que la ven como una herramienta para fortalecer la continuidad de las em-

3 Existen casos como el de la ERT Mecber que fabricaba autopartes para automotrices, estos trabajadores se vieron obligados a conformar una sociedad comercial, a la par de la cooperativa, ya que sus proveedores por normas internas, no le compraban a cooperativas. 
presas en contraste con los que plantean la rápida liquidación del activo falencial, en aras de no continuar perjudicando el crédito comercial.

\section{Concepto de ERT desde un enfoque jurídico}

Ahora bien, definida la cuestión social y el mecanismo que establece la LCQ, conforme se erige el fenómeno, han surgido una batería de normas jurídicas que han intentado regular desde diversas miradas y enfoques a las ERT.

Para relevar la definición desde una orientación jurídica, se procedió a realizar un trabajo de geolocalización y sistematización de normas sancionadas que hicieran mención al descriptor «empresa recuperada». De este trabajo se llegó a la conclusión de que a nivel federal no existe hasta la fecha ${ }^{4}$ una definición o categorización jurídica sobre lo que es una ERT. No obstante ello, en función a la tarea descripta se han relevado múltiples referencias y hasta definiciones en normativas locales y menciones en normativas de alcance federal.

En la siguiente tabla se pueden verificar la cantidad de normas a nivel federal, provincial y municipal donde se denominan a las Empresas Recuperadas. La tabla fue realizada teniendo en cuenta el tipo de norma de que se trata y el año de su emisión:

Como se desprende del cuadro anterior, el año 2020 concentra el $20 \%$ del total de las normas relevadas, pero es dable mencionar que este incremento se debe simplemente a que en el contexto de medidas para paliar la crisis del virus SARS-COVID 2019, el Estado Nacional dictó un Decreto con una serie de medidas para la protección de diversos sectores sociales y económicos, entre dichas medidas se encuentra una relacionada con los inmuebles ocupados por «Empresas Recuperadas» de allí la primera aparición del término en el año. A su vez, el decreto nacional invitaba en el mismo acto a adherir a las provincias a dichas medidas, hecho que muchas realizaron replicando a nivel provincial los beneficiarios de la norma nacional de manera textual. Lo propio hicieron diversos organismos estatales con competencia federal como el ENARGAS, el Ministerio de Desarrollo Productivo, entre otros, por lo que ese incremento se explica por dicha particularidad.

4 Si bien existe un proyecto de ley nacional de empresas recuperadas presentado en noviembre de 2020, hasta la fecha de redacción del presente artículo el mismo no ha tenido tratamiento. 


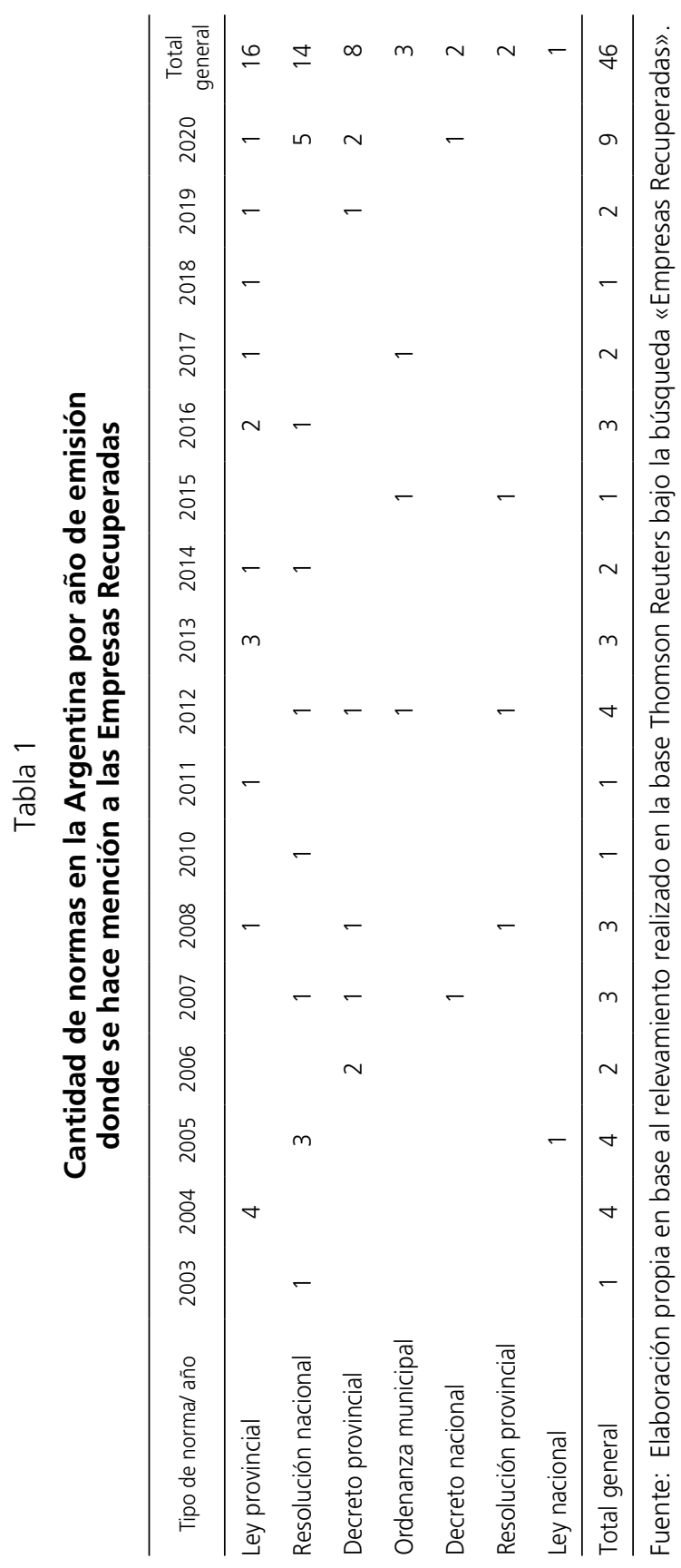


Otra cuestión para destacar es que los mayores tipos de normas relevadas donde se mencionan a las ERTs son leyes provinciales de 7 jurisdicciones distintas, a saber: Córdoba con 4 leyes; Río Negro y la Ciudad Autónoma de Buenos Aires con 3 cada una; Santa Fe y la Provincia de Buenos Aires con 2 cada una y; por último Chubut y Mendoza con una norma cada una. De esto se desprenden varias conclusiones preliminares, por un lado, que más del $29 \%$ de las jurisdicciones del país, cuentan con leyes específicas referidas a este fenómeno, encontrándose entre ellas las provincias más pobladas de la Argentina, y que por otro lado son los territorios del país con mayor industrialización como el caso de Córdoba, la Ciudad Autónoma de Buenos Aires, la Provincia de Buenos Aires y Santa Fe.

En lo que refiere a la definición de ERT, de las 46 piezas relevadas, sólo en 8 de ellas se las caracteriza, pero ésta se realiza de una manera no uniforme tal como se verá a continuación.

Es así como se encuentran normas que limitan la calidad de ERT a tener una ley que declara la utilidad pública de algún bien sujeto a expropiación, como es el caso de la normativa emanada de la Ciudad Autónoma de Buenos Aires en el año 2004, la ley de la Provincia de Buenos Aires en el año 2008 o la Ordenanza dictada en el año 2012 en el Municipio de San Martín. También se ha relevado un caso con una definición mas amplia y por ende menos restrictiva, en la que se requiere únicamente que la gestión se encuentre en manos de sus trabajadores, como es el Decreto de la Provincia de Buenos Aires del año 2012, el que las define como "a las unidades económicas productivas y/o de servicios que, sin importar la figura jurídica bajo la cual se hallen organizadas, son gestionadas directamente por sus trabajadores».

Pero la corriente mayoritaria, a nivel normativo, limita el concepto a la forma jurídica de Cooperativa de Trabajo, y esta asimilación se encuentra en variadas jurisdicciones con la particularidad de que comienzan a aparecer desde el año 2013 en adelante, tal como se puede observar en la siguiente tabla realizada en base al mapeo mencionado. En esta solo se exhiben aquellas piezas normativas donde se define a una ERT: 


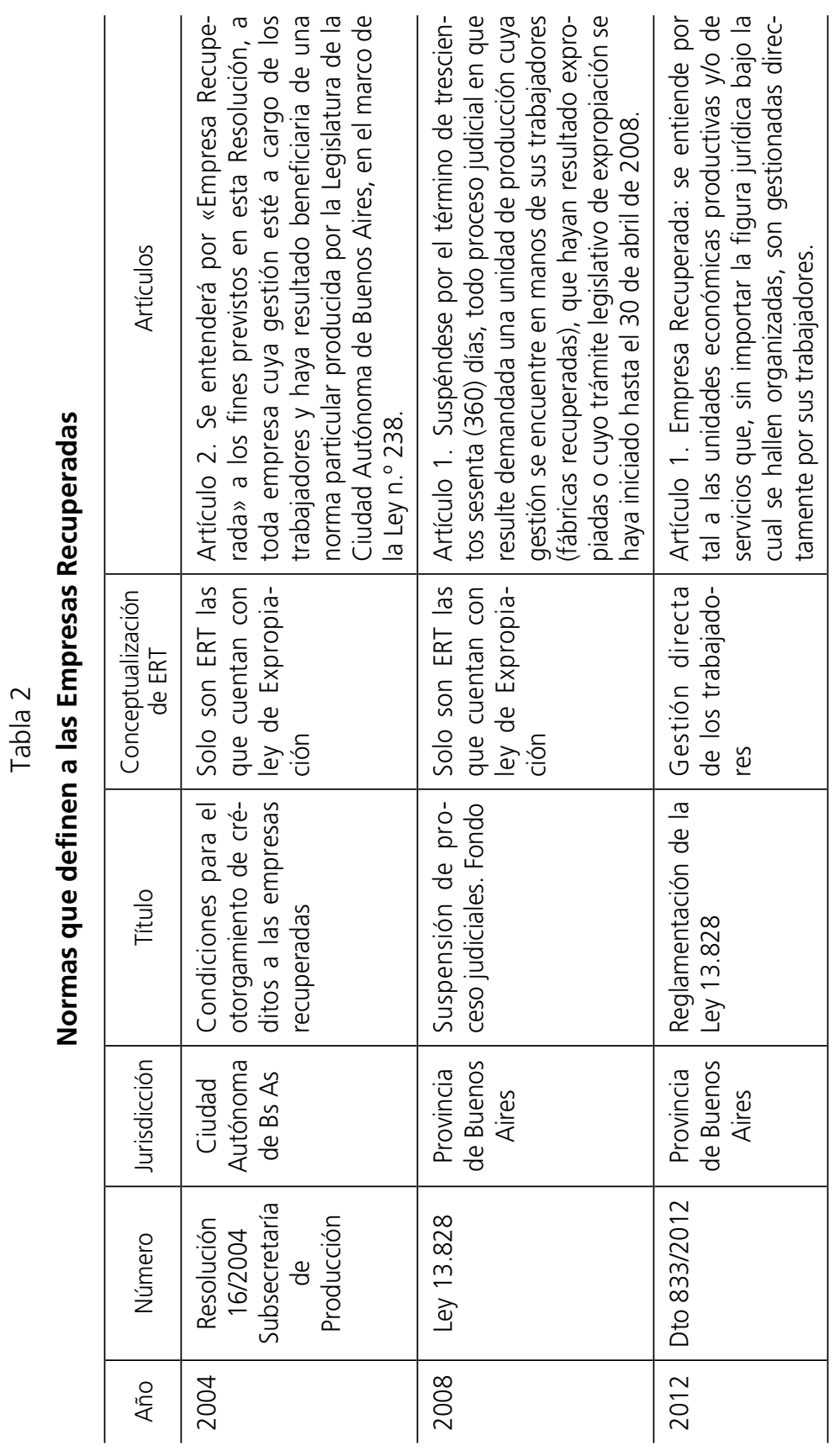

Deusto Estudios Cooperativos

ISSN: 2255-3444 • ISSN-e: 2255-3452, Núm. 18 (2021), Bilbao, pp. 149-176 


\begin{tabular}{|c|c|c|}
\hline$\frac{\tilde{O}}{\frac{0}{3}}$ & 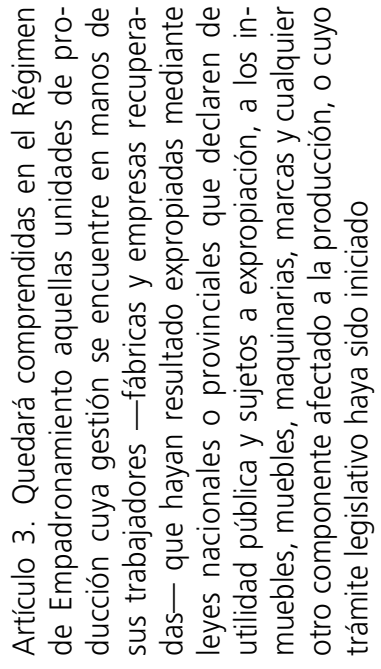 & 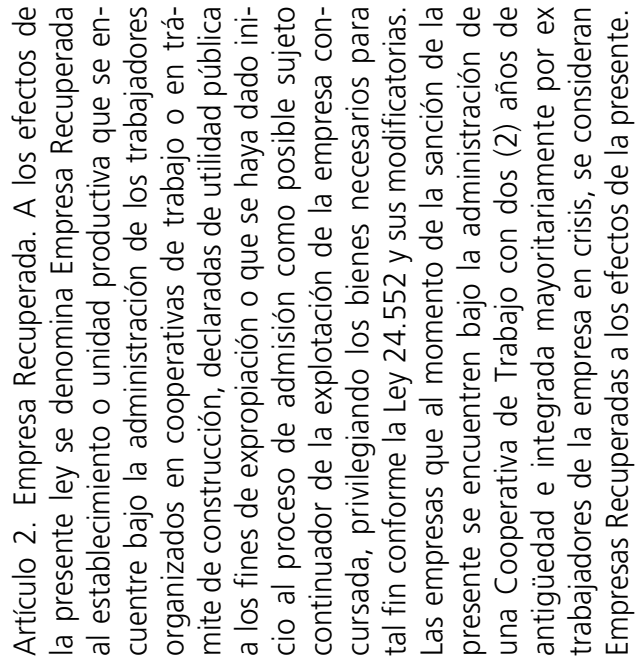 \\
\hline 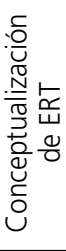 & 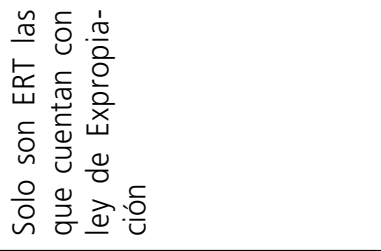 & 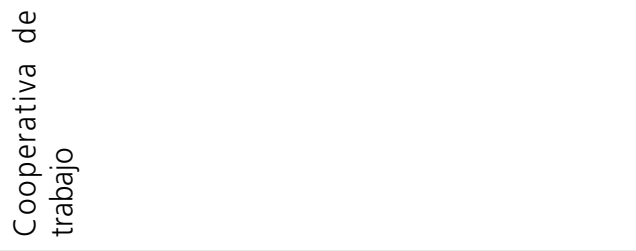 \\
\hline$\stackrel{\text { 을 }}{\stackrel{ \pm}{E}}$ & 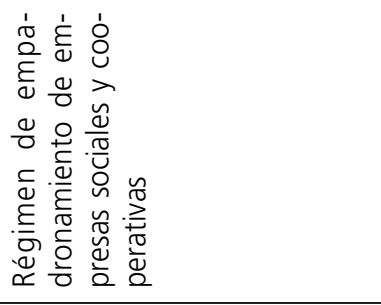 & 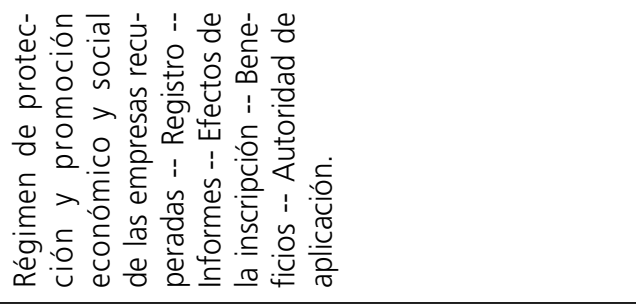 \\
\hline 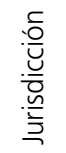 & 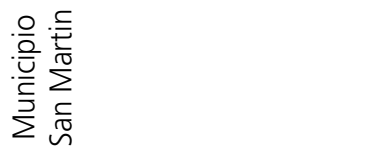 & $\begin{array}{l}\text { 음 } \\
\text { d) } \\
\frac{0}{1}\end{array}$ \\
\hline 这 & 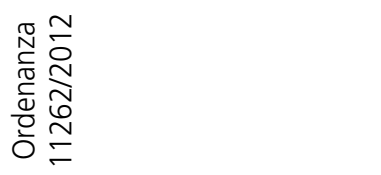 & 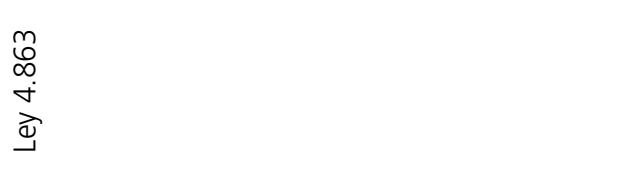 \\
\hline 号 & $\frac{\sim}{\stackrel{\sim}{\sim}}$ & \\
\hline
\end{tabular}




\begin{tabular}{|c|c|}
\hline$\frac{\text { O }}{\frac{U}{3}}$ & 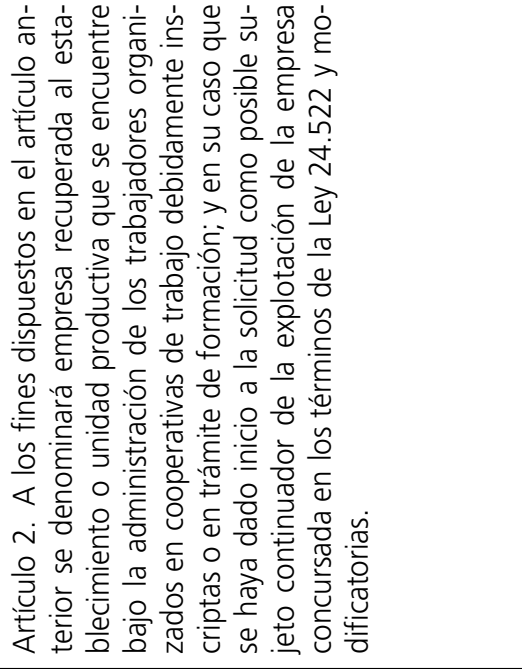 \\
\hline 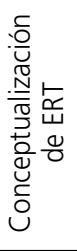 & 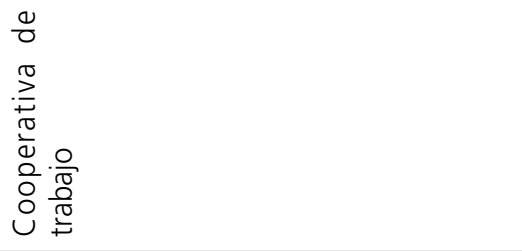 \\
\hline$\underset{i}{\stackrel{O}{D}}$ & 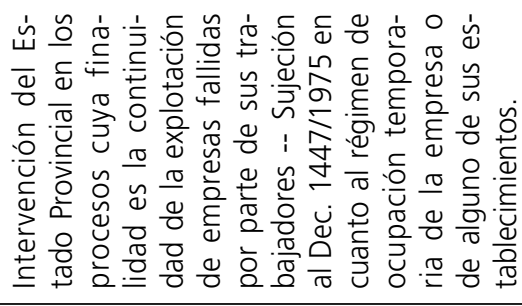 \\
\hline 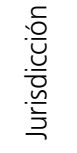 & 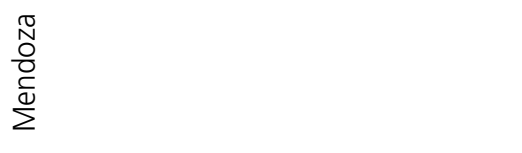 \\
\hline $\begin{array}{l}\text { O } \\
\text { E } \\
\text { ¿. }\end{array}$ & 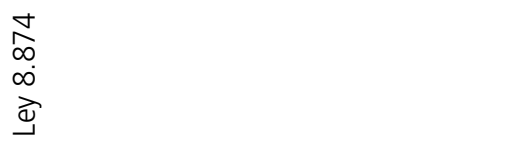 \\
\hline 号 & 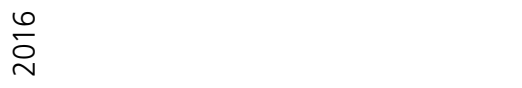 \\
\hline
\end{tabular}




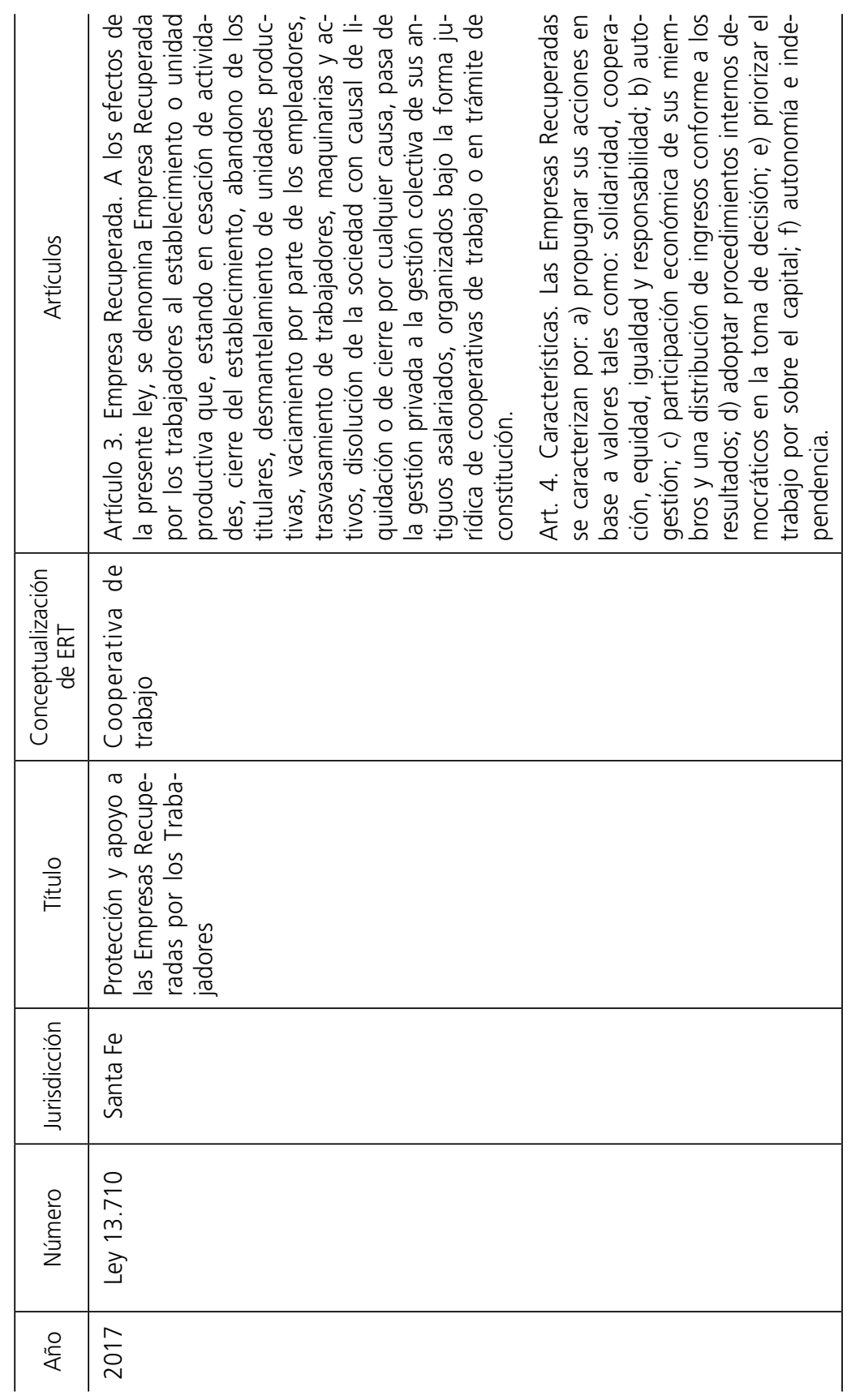




\begin{tabular}{|c|c|}
\hline 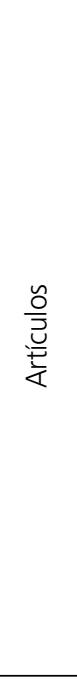 & 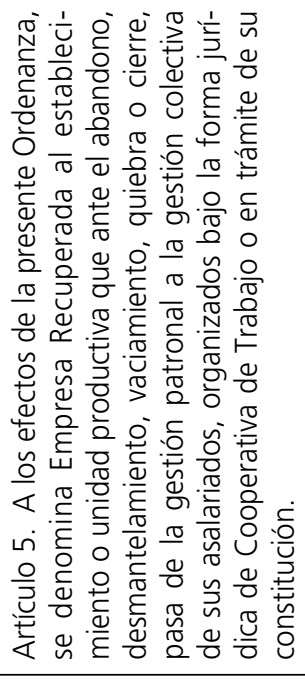 \\
\hline 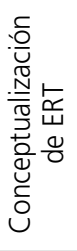 & 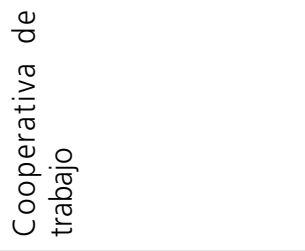 \\
\hline 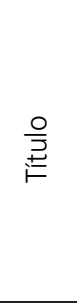 & 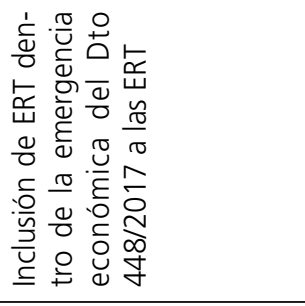 \\
\hline 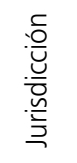 & 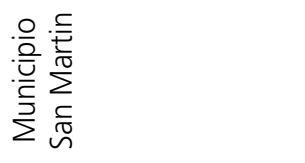 \\
\hline 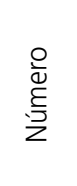 & 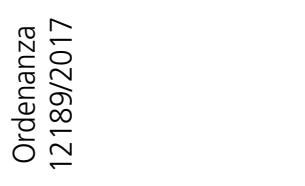 \\
\hline 产 & $\frac{N}{\circ}$ \\
\hline
\end{tabular}


Un caso testigo de la anomia normativa, se destaca en una jurisdicción, en este caso el Municipio de San Martín, el que define a las ERTs de dos maneras diferentes e incongruentes. Es así como en el año 2012 para ser considerada dentro de esa categoría se exigía contar con una ley que declara la utilidad pública y sujeto a expropiación de algún bien de la fallida sin importar la forma jurídica y en 2017, 5 años luego, el criterio de inclusión cambió para ser uno de los aspectos centrales la forma jurídica de cooperativa de trabajo.

No obstante lo mencionado, se ve de esta manera como existe una tendencia a asimilar y cerrar el concepto de ERT a una forma jurídica particular, la cooperativa de trabajo, en consonancia con la Ley de Concursos y Quiebras y las características comunes mencionadas.

\section{Particularidades en materia legal, fiscal y de la seguridad social}

Es así como la centralidad del cooperativismo en los procesos de recuperación de empresas ha generado una adaptación desde ambos lados (tanto de las experiencias como del movimiento cooperativo), ya sea mediante el cumplimiento de las regulaciones generales que pesan sobre todo el universo de entidades enmarcadas en el Instituto Nacional de Asociativismo y Economía Social (en adelante INAES), así como por la integración de éstas a diversas federaciones de cooperativas como ser la Federación de Cooperativas de Trabajo (Fecootra), la Federación de Cooperativas de Trabajadores Autogestionados (Facta) o la Red Gráfica Cooperativa, entre otras.

En este apartado, analizaremos entonces como la normativa cooperativa, ha recepcionado las experiencias de recuperación de empresas por cooperativas en materia de control, financiamiento, tributación y seguridad social.

\subsection{Organismo estatal de promoción y control. Datos desde el Estado}

Como hemos visto, la conceptualización como cooperativa de trabajo, lleva consigo una diversidad de encuadres que no siempre son armónicos con la realidad particular de este colectivo (Pocztaruk, 2004).

Es así como en materia societaria, su organismo de promoción y fiscalización, es el INAES dependiente del Ministerio de Desarrollo Productivo. Este Instituto creo en el año 2020 un registro de consulta pú- 
blica ${ }^{5}$, en donde se detallan las distintas experiencias, su ubicación geográfica, actividad en la que se desempeñan y la cantidad de trabajadores con que cuentan'

De dicho registro, se desprende que existen a la fecha para la autoridad de aplicación 426 ERTs.

Es curioso mencionar que en la literatura se suele relacionar a las ERTs con el año 2000 y 2001, pero del análisis del mencionado registro se desprende que 13 entidades que representan un 3\% del total de recuperaciones se realizaron con anterioridad. Es así como se registran 4 recuperaciones en la década del 50, 1 en 1972 y 18 en la década del 90.

Respecto a la distribución geográfica es representativa la concentración en la zona del Área Metropolitana de Buenos Aires, que comprende a la Ciudad Autónoma de Buenos Aires y a la provincia de Buenos Aires delimitadas por cordones suburbanos y conurbanos. En el siguiente gráfico se puede observar lo mencionado en el gráfico 1.

5 Desde el Ministerio de Desarrollo Social de la Nación existiría otro registro denominado Registro Nacional de Empresas Recuperadas (RENACER) el cual ha sido organizado por la Dirección de Empresas Recuperadas, cuyos datos, conformación y particularidades de inclusión, hasta la fecha de la redacción del presente artículo no son públicos.

6 Este registro es de consulta pública y puede visualizarse en el siguiente enlace https://www.argentina.gob.ar/inaes/registro-nacional-de-empresas-recuperadas (último acceso en junio 2021) 


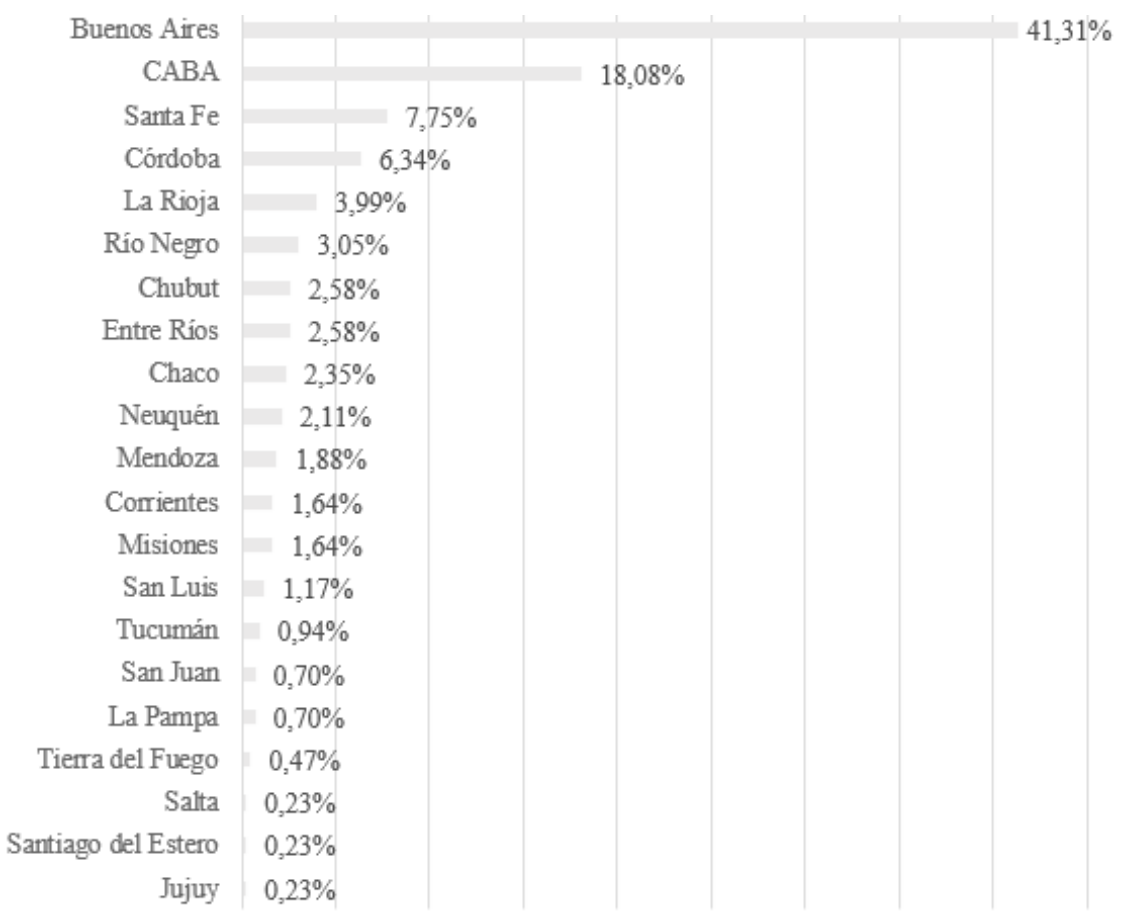

Fuente: elaborado por Leonardo Chiesa en función a la base de ERT del INAES (acceso junio 2021).

\section{Gráfico 1}

\section{Distribución de ERT por provincia}

Sumado a ello se estima en dicho registro que en la actualidad existen 15.963 trabajadores enmarcados en las ERT. 
Por último, otro dato relevante que surge del registro es la distribución por actividad económica, siendo la rama de industria manufacturada como la mayor proporción tal como se puede apreciar en el siguiente gráfico:

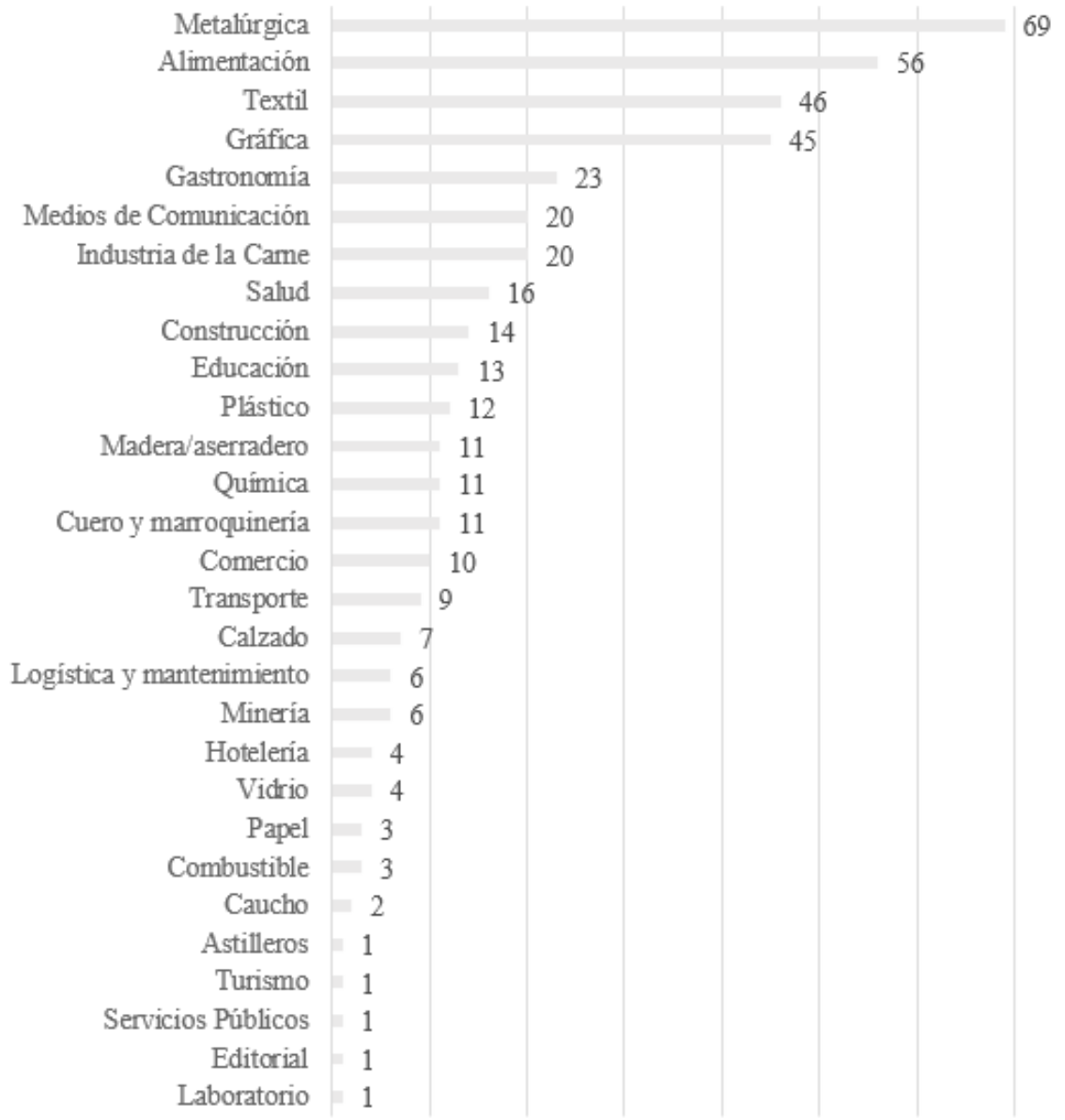

Fuente: elaboración propia en función a la base de ERT del INAES (acceso junio 2021).

Gráfico 2

\section{Cantidad de ERTs por sector de actividad}


A pesar del surgimiento de este registro, no se ha creado hasta la fecha una dependencia formal dentro del INAES que atienda las demandas del sector, aunque en el año 2020 se constituyó una Comisión Técnica Asesora de "Empresas Recuperadas», que fue la encargada de confeccionar la sistematización antes mencionada.

Cierto es que el Estado se define por acción u omisión, en este caso, al no haber una dependencia formal del Instituto, las políticas públicas que lleva el organismo en términos generales, (ya que hemos visto algunas particularidades con respecto a la comisión, al registro y veremos a continuación una línea de financiamiento particular) son uniformes tanto para entidades donde los asociados decidieron conformarse en cooperativas por la propia voluntad como aquellos grupos que deben hacer uso de esta figura en el marco de un proceso falencial donde los tiempos son impuestos por una normativa externa, la LCQ y por una necesidad apremiante, continuar con la explotación para generar recursos. Por lo tanto, la Cooperativa de Trabajo que deviene de un proceso falencial surge de un punto de partida diferencial, caracterizado por la escasez de capital para reanudar la producción o la prestación de servicios, deudas ocasionadas por la fallida, las problemáticas enmarcadas en el propio proceso judicial, la situación respecto a los proveedores de materia prima, de servicios públicos y la dificultad de afianzar clientes que por lo general se han visto perjudicados, entre otros elementos que deben ser abordados por políticas públicas diseñadas para resolver tal escenario en un tratamiento diferencial, hecho que en la actualidad no sucede de manera integral.

\subsection{Financiamiento}

En materia de acompañamiento económico y financiero por parte del Estado, se destaca a nivel nacional la creación en el año 2004 del Programa Trabajo Autogestionado dependiente del Ministerio de Trabajo y Seguridad Social de la Nación. Este programa fue creado con apoyo de las Naciones Unidas para el Desarrollo mediante el Proyecto PNUD ARG/02/03 y se pensó en sus inicios en función a las particularidades de las ERT, diseñando así distintas líneas de financiamiento según el momento del proceso en que se encontrara la entidad. Es así como la línea 1 se encontraba destinada a reforzar los ingresos individuales de los trabajadores mediante una ayuda económica individual, imprescindible en los inicios de estas experiencias donde los ex empleados de la fallida se encuentran en pleno conflicto, con la producción muchas veces parada y por ende sin ingresos. La línea 2 destinada a 
financiar principalmente capital de trabajo, y las siguientes líneas relacionadas a apoyo técnico y económico para la mejora de la capacidad productiva, la competitividad, gestión y asistencia para la higiene y seguridad del trabajo.

A su vez, el INAES contaba en el año 2011 con una línea específica de financiamiento para atender las demandas de este sector, el que en la actualidad se encuentra derogado7. La particularidad que tenían estos préstamos con tasas de interés preferenciales es que era el único a nivel nacional que permitía de manera explícita y directa, utilizar los recursos para contribuir a la regularización patrimonial de estas experiencias, incluyendo entre estos la compra de bienes a la quiebra, así como inmuebles para evitar posibles desalojos propios de procesos que conllevan en su génesis la liquidación de los bienes mientras los trabajadores intentan generar los recursos para obtenerlos.

Como se puede apreciar y se verá en el punto 4.3, los fondos que el INAES ponía a disposición de las ERT provenían del propio sector cooperativo, por lo que se vislumbra de esta manera como era el cooperativismo el que colaboraba con el afianzamiento de otras experiencias, materializando de esta manera el principio de ayuda mutua.

En lo que refiere al ámbito local, la Provincia de Buenos Aires creó ${ }^{8}$ primero en el año 2007 un fideicomiso de asistencia financiera a empresas recuperadas (denominado FAFERBA), el que incluso reglamentó en el mismo año. A su vez, en el año 2008 avanzó en un Fondo Especial de Recuperación de Fábricas de la Provincia de Buenos Aires ${ }^{9}$, el que además fue reglamentado mediante un decreto que ampliaba el concepto de ERT, creaba un Consejo Asesor y reglamenta todos los pasos para hacerse operativo. No obstante toda la normativa relevada sobre estos dos instrumentos, hasta la fecha, se desconoce si dicho fondo se ha aplicado a alguna ERT en la Provincia de Buenos Aires.

\subsection{Regulación fiscal}

En lo que refiere al ámbito fiscal, a nivel federal cuentan con la misma regulación que una cooperativa de trabajo, no existiendo un tratamiento específico que dé cuenta de su particular historicidad y características.

\footnotetext{
7 Se trataba de la Resolución 4156/2020 emitida por el INAES el 29/12/2010.

8 Mediante Decreto 86/2007 y luego Reglamento con el Decreto 2916/2007.

9 Mediante la Ley 13.828.
} 
Es así cómo deben tributar el Impuesto al Valor Agregado (IVA), cuya alícuota y exenciones depende de las actividades que realice, siendo la general del impuesto, la del $21 \%$.

A su vez, recae sobre estas la obligación de inscripción y posterior tramitación de la exención en el Impuesto a las Ganancias. Esta situación de exención y no exclusión del tributo, se debe a un incorrecto encuadre histórico que sufren las cooperativas en la Argentina y que el movimiento cooperativo reclama incesantemente.

Es así como encuadrar a las entidades de manera incorrecta genera dificultades no solo conceptuales sino también administrativas, ya que se les exige atravesar un proceso por el cual la administración fiscal, denominada Administración Federal de Ingresos Brutos (AFIP) realiza un análisis de la forma jurídica, y en algunos casos de los movimientos financieros internos de la entidad para otorgarla. La exención en la actualidad tiene un plazo de duración determinado y su renovación requiere además de una certificación contable específica, que conlleva gastos de honorarios al profesional certificante así como pagos al Consejo Profesional. A su vez, en el caso de que esta no sea renovada o la entidad omitiera directamente tramitarla, el Fisco entiende que recae sobre la entidad el impuesto por los períodos donde esta no se encontraba vigente, por lo que se ha intentado, desde el organismo recaudatorio, aplicar la alícuota del 35\% sobre el resultado de los ejercicios de la cooperativa.

Además las entidades se encuentran sujetas, como todas las cooperativas, a la Contribución Especial sobre el Capital Cooperativo, denominado por el fisco como FONEDU. Éste se abona en anticipos en función al capital cooperativo calculado sobre cierta porción del patrimonio de la entidad.

En el año 1986, cuando se creó la norma, se pensó en una contribución transitoria que formaría parte de un fondo para fortalecer al sector cooperativo. Es así como lo recaudado por el fisco corresponde en un $75 \%$ al INAES y un $25 \%$ se distribuye entre las provincias en función al régimen de coparticipación federal.

En los debates dados en el seno del Congreso de la Nación al momento de crearlo (Feser, 2017), se planteó si dicha distribución provincial era la más adecuada, teniendo en cuenta que en su diseño se trata de recursos que se destinan a un fondo para la promoción del cooperativismo y el régimen de distribución provincial corresponde a otros parámetros que no se relacionan con la densidad de cooperativas en cada territorio sino que es producto del acuerdo político al que arribaron las provincias para distribuirse recursos fiscales de otra naturaleza. A esto se suma la dificultad de que el monto coparticipable no tiene un des- 
tino específico como sí lo tiene el correspondiente al INAES, a quien la norma le demanda al organismo aplicarlo a ciertas actividades para fortalecer al sector cooperativo, entre ellas se encuentra la línea de financiamiento analizada en el punto 4.2 específica para ERTs.

En el ámbito provincial, existen diversos tributos locales, cuya regulación depende de cada jurisdicción, por lo que el hecho imponible se genera, según el caso, si la entidad se encuentra radicada en ella o realiza ciertas actividades allí. En lo que refiere a ERTs, del relevamiento mencionado en los apartados anteriores, se han encontrado 16 leyes provinciales, pero solo 3 otorgan exenciones específicas para las ERTs. Estas son las provincias de Santa Fe, Córdoba y la Ciudad Autónoma de Buenos Aires, con la particularidad de que en esta última se exigía un certificado de ERT que ningún organismo de la Ciudad otorgaba, por lo que se tornó de imposible cumplimiento.

\subsection{Seguridad social}

En el ámbito de la seguridad social, es donde las ERTs encuentran mayores dificultades para reconocerse dentro del cooperativismo de trabajo toda vez que en la actualidad el sistema no tiene en cuenta las particularidades del trabajo autogestionado.

La regulación actual es producto de un proceso particular, que encuentra tres hitos producidos en los años 1992, 1998 y 2013.

En el primero de ellos, del año 1992, se sancionaron dos resoluciones, una por parte del organismo de control de las cooperativas ${ }^{10}$ y otra por la ANSES ${ }^{11}$, el organismo estatal que se encarga de administrar los fondos relacionados a la seguridad social en el país. Ambas resoluciones consideraban que los trabajadores de cooperativas de trabajo, que hasta ese momento cotizaban a la seguridad social como trabajadores bajo relación de dependencia, podían hacerlo ahora como autónomos, pero esta última categoría era obligatoria para los nuevos trabajadores. Este cambio generaba una modificación sustancial en los componentes de la seguridad social, como veremos más adelante.

El segundo hito se produce en el año 1998 cuando se crea en el país un régimen denominado monotributo, que simplifica en un solo monto impuestos nacionales, contribuciones relacionadas con la jubilación y aportes a una obra social. Es así como los trabajadores de coo-

10 Resolución INAC 183/92.

11 Resolución ANSES 784/92. 
perativas de trabajo contaban ahora con dos posibilidades, o ser autónomos sin topes de ingresos o entrar en el régimen del monotributo, el cual tenía la limitante de ingresos anuales.

Finalmente en el año 2013, el INAES dicta una Resolución ${ }^{12}$ que amplía el abanico de posibilidades, ahora podrían además elegir la opción de cotizar como trabajadores en relación de dependencia, de esta manera se completaba así el abanico de opciones con que se cuenta en la actualidad.

Las diferencias entre los tres sistemas deben ser analizadas desde dos perspectivas, por un lado los costos que tiene y por el otro los cinco componentes de la seguridad social en la Argentina, los cuales son 1) Asignaciones familiares; 2) Aseguradora de Riesgos del Trabajo (ART); 3) Obra social; 4) Seguro de desempleo; y 5) Jubilación.

En lo que hace a la primera perspectiva, vale mencionar que administrativamente el monotributo es el más simple ya que se trata del pago de un monto por asociado de manera mensual que incluso se puede realizar mediante una declaración jurada que contemple a la totalidad de los asociados. En lo que refiere a autónomos, en principio es similar al monotributo pero en términos económicos es más caro que éste, con el agravante que cada asociado deberá anualmente realizar una declaración jurada en el impuesto a las ganancias, tornando la categoría de autónomos no solo más cara en términos económicos sino también en términos administrativos.

A su vez estas dos categorías cuentan con problemas relacionados a que entienden a los trabajadores de manera individual y no como un colectivo que se autogestiona, por lo que parte de las críticas que se realizan desde el sector de las ERTs y el cooperativismo de trabajo en general se encuentran relacionadas con ser una categoría tributaria y de la seguridad social que no contempla las particularidades del trabajo asociado.

En lo que refiere a la categoría de los dependientes, vale mencionar que si bien es el encuadre que los trabajadores de ERTs solían tener antes de la recuperación de la empresa, es administrativamente más compleja que las otras porque requiere de la presentación de una declaración jurada mensual y el cálculo de retenciones en el impuesto a las ganancias (si corresponde por los montos de las retribuciones). A esto se suma un costo mayor que el resto de las posibilidades ya que entre los aportes realizados por los trabajadores y las contribuciones que debe realizar la cooperativa se estima que rondan el $47 \%$ del monto

12 Resolución 4664/2013. 
a percibir por el asociado, porcentaje ostensiblemente mayor que los otros dos regímenes. Además, el encuadre tiene dificultades de índole relacionales, toda vez que se ve a la cooperativa como un empleador y a los trabajadores como dependientes de ésta, cuando en los hechos no es este el vínculo real que prevalece en una forma de organización cooperativa donde el eje central es el trabajo de sus asociados.

En lo que refiere a la segunda perspectiva, a saber un análisis desde los cinco componentes de la seguridad social, allí también se verifican diferencias entre los tres regímenes además de particularidades relacionadas con las ERTs.

Es así como en lo que refiere al primer componente, el de las asignaciones familiares, la elección de los regímenes del monotributo o autónomos, carecen del amplio abanico que contempla el encuadre como trabajador dependiente.

En lo que refiere a riesgos del trabajo, ocurre algo similar, teniendo que optar los asociados a cooperativas de trabajo por seguros de accidentes personales si optan por encuadrarse dentro del monotributo o autónomos. Estos seguros además, no cubren las mismas fatalidades que una ART de un trabajador dependiente.

El tercer componente vinculado a la obra social encuentra dificultades ya que los aportes que se realizan en el monotributo son de muy bajo monto por lo que ciertas obras sociales dificultan la atención de sus afiliados sumado a que la incorporación del grupo familiar dentro del plan genera un costo adicional, en contraposición con la categoría de los dependientes donde la cobertura se realiza sobre todo el grupo familiar, con independencia de los aportes realizados por el trabajador.

Sobre el seguro de desempleo, en el caso donde solo existe cobertura es si se opta por el encuadre como trabajador dependiente.

Finalmente en lo que refiere a la jubilación, tanto la categoría de autónomo como la de monotributo generan un mínimo de aporte que provoca que los trabajadores se jubilen con el haber mínimo, en contraposición con el sistema de los dependientes donde la jubilación se calcula en base al promedio de los últimos diez años de aportes. Referido a este punto las ERTs suelen tener el inconveniente para los socios fundadores, quienes ven licuar sus aportes realizados cuando eran empleados de la ahora empresa recuperada cada año que transcurren bajo la figura cooperativa, generándose incluso situaciones donde aquellos trabajadores que cuentan con los años de aporte mínimos para jubilarse pero no con la edad requerida, prefieren no encontrarse registrados en la seguridad social bajo la figura del monotributo a la espera de cumplir la edad y tramitar su jubilación como ex empleado de la firma quebrada. 


\section{Reflexiones finales}

Como hemos visto a lo largo de este breve artículo, el cooperativismo ha sido un espacio de acompañamiento a experiencias de recuperación de empresas. Este acompañamiento se ha visto de alguna manera traccionado no solo por la normativa concursal sino también por ser la única figura de la totalidad existente que cumple con varias de las demandas del colectivo en esta nueva etapa de autogestión, donde la solidaridad, la ayuda mutua, el principio de un socio un voto, confluyen a la perfección con la cooperativa de trabajo.

Hemos visto también como no existe desde la normativa relevada definiciones unívocas sobre lo que es una ERT, pero de las pocas halladas la tendencia mayoritaria desde el año 2013 a la fecha es caracterizarlas como cooperativas de trabajo, cerrando por un lado toda posibilidad de utilización de otra figura pero al mismo tiempo reconociendo en el cooperativismo una identidad particular.

A su vez, el propio sector cooperativo se ha encargado mediante el INAES de promover líneas de financiamiento con recursos de la Contribución Especial sobre el Capital Cooperativo, abonando así la idea de ayuda mutua para colaborar con el fortalecimiento de estas experiencias en las necesidades particulares de adquirir ciertos bienes de la quiebra, que no suelen ser financiables ni por bancos ni por otras líneas del Estado que aunque atienden a necesidades específicas de los procesos de recuperación de empresas no llegan a cubrir todas las necesidades del colectivo en un proceso falencial.

En este sentido hemos analizado también como otros programas estatales se han diseñado para acompañar a estas experiencias y sus especificidades. Podemos mencionar entre estas la línea 1 del Programa Trabajo Autogestionado, que brinda una ayuda económica a cada trabajador mientras espera que los tribunales le otorguen la posibilidad de trabajar o en los primeros meses de la actividad bajo forma cooperativa.

Desde la esfera fiscal, se han relevado diversos problemas con los encuadres, algunos compartidos con otras ramas del cooperativismo como es la exención y no la exclusión en el impuesto a las ganancias, otras más específicas del cooperativismo de trabajo en su conjunto como son los problemas derivados de la seguridad social y finalmente el referido a la jubilación que impacta directamente en las experiencias de ERTs, por lo que resta mucho en este campo por avanzar para lograr una protección integral de estas experiencias en su camino por fortalecer el trabajo autogestionado cooperativo. 
En definitiva, el modelo de ERT se erige y persiste pese a las dificultades que atraviesan mencionadas a lo largo del trabajo, seguramente debido a diversos factores entre los cuales, los valores de la solidaridad y ayuda mutua poseen un lugar central.

\section{Referencias}

CorAggio, José Luis: «La Presencia de la Economía Social y Solidaria y su Institucionalización en América Latina», en Instituto de Investigación de las Naciones Unidas para el Desarrollo Social, Documento Ocasional 7, para el Proyecto Potencial y Límites de la Economía Social y Solidaria, Suiza, pp. 1-24, 2014. Disponible en: http://www.unrisd.org/80256B3C005BCCF9/\%28htt pAuxPages\%29/7A2E9D246F8B55B2C1257CF900428C90/ \$file/OP7Coraggio.pdf

Chiesa, Leonardo; Godoy Pablo; Navaza y LiéBAnA, Pedro: «Ley de Promoción de la Economía Social en la Ciudad Autónoma de Buenos Aires. Objetivos, alcances, precisiones y desafíos. Revista Idelcoop, No 233, ISSN Electrónico 2451-5418 / P. 210-228 / Sección: Normativa

Dubois FAVIER (h.) E.M.: Reglamentaciones necesarias para la correcta aplicación de la reforma de la ley de quiebras. AR/DOC/2433/2011. Recuperado de Sistema de Información Legal. 2011

FARH DE MONTALBÁN, D.: La recuperación de empresas fallidas por sus trabajadores. El proyecto de reformas. Algunas propuestas. El Derecho Diario. 212, (799). ED-DCCLXVII-665. Recuperado de Editorial El Derecho. 2005.

FESER, M.: La contribución especial sobre el capital cooperativo (FONEDU). Entre el fortalecimiento del sector y el desincentivo a invertir. Trabajo Final, Maestría en Derecho y Economía. Universidad Torcuato Di Tella. 2017. Disponible en https://repositorio.utdt.edu/bitstream/handle/utdt/11198/ MDT_2017_Fesser.pdf? sequence=1\&isAllowed=y

JUNYENT BAS, F.: La reforma del ordenamiento concursal introducida por la ley 26.684(1). El Derecho Diario. 243, (991). ED-DCCLXXII-384. Recuperado de Editorial El Derecho.2011

PoczTARUK, E.: Marco legal y viabilidad de las empresas recuperadas: «el día después». VI Jornadas de Sociología. Facultad de Ciencias Sociales, Universidad de Buenos Aires, Buenos Aires. 2004

Programa FACULTAD ABierta: "Primer informe del relevamiento entre empresas recuperadas por los trabajadores», en Programa Facultad Abierta Secretaría de Extensión Universitaria Facultad de Filosofía y Letras Universidad de Buenos Aires, 2014. disponible en: https://www.recuperadasdoc.com.ar/Informes\%20relevamientos/Informe\%20Primer\%20relevamiento\%202003.pdf

Programa FACULTAd AbIeRTA: «Informe del IV Relevamiento de Empresas Recuperadas en la Argentina». Facultad de Filosofía y Letras, Universidad de Buenos Aires. Buenos Aires, Argentina, 2014. 
REBÓN, J.: La Empresa de la Autonomía. Trabajadores recuperando la producción. Buenos Aires, Picaso. 2007

MAFFIA, O.J.: Otra oportunidad perdida: más de lo mismo en materia concursal. Revista La Ley. 156, 1. Id SAIJ: DACA950147. 1995. Recuperado de http:// www.saij.gob.ar

TÉVEZ, A.N.: La cooperativa de trabajo como continuadora de la empresa en quiebra. AR/DOC/2372/2011. Recuperado de: http://www.derecho.uba.ar/ institucional/pacem/pdf/la-cooperativa-de-trabajo-como-continuadora-dela-empresa-en-quiebra.pdf

\section{Normativa}

DECRETO 1951/2007.

DECRETO 311/2020.

LEY 24.522, de Concursos y Quiebras. Disponible en http://servicios.infoleg. gob.ar/infoleglnternet/anexos/25000-29999/25379/texact.htm

LEY 26.058.

LEY 26.684. Reforma a la Ley de Concursos y Quiebras. Disponible enhttp:// servicios.infoleg.gob.ar/infoleglnternet/anexos/180000-184999/183856/ norma.htm

RESOLUCIÓN 4156/2010 INAES.

RESOLUCIÓN ANSES 784/92. Disponible enhttps://www.argentina.gob.ar/normativa/nacional/resoluci\%C3\%B3n-784-1992-27797

RESOLUCIÓN INAC 183/92. Disponible enhttps://vpo3.inaes.gob.ar/files/resoluciones/inac/rinac183_92.pdf

RESOLUCIÓN INAES 319/2020.

RESOLUCIÓN INAES 4664/2013. Disponible enhttp://servicios.infoleg.gob.ar/infoleglnternet/anexos/220000-224999/224801/norma.htm 


\section{Derechos de autor}

La revista Deusto Estudios Cooperativos es una revista de acceso abierto lo que significa que es de libre acceso en su integridad inmediatamente después de la publicación de cada número. Se permite su lectura, la búsqueda, descarga, distribución y reutilización legal en cualquier tipo de soporte sólo para fines no comerciales y según lo previsto por la ley; sin la previa autorización de la Editorial (Universidad de Deusto) o el autor, siempre que la obra original sea debidamente citada (número, año, páginas y DOI si procede) y cualquier cambio en el original esté claramente indicado.

\section{Copyright}

The Deusto Journal of Cooperative Studies is an Open Access journal which means that it is free for full and immediate access, reading, search, download, distribution, and lawful reuse in any medium only for non-commercial purposes, without prior permission from the Publisher or the author; provided the original work is properly cited and any changes to the original are clearly indicated. 